\title{
Multiple Users Round Trip Time Models in IEEE 802.11b WLANS
}

\section{OGHOGHO, I}

\author{
Department of Electrical/Electronic Engineering, Delta State University, Abraka, Delta State, Nigeria. \\ Corresponding Author's Email: oghogho.ikponmwosa@delsu.edu.ng,oghoghoik@gmail.com \\ Tel: +2348060676748
}

\begin{abstract}
The dependence of Round Trip Time (RTT) on Signal to Noise Ratio (SNR) for multiple users in an IEEE 802.11b Wireless Local Area Network (WLAN) was studied in this work. Data collected in open corridor, small offices and free space environments in an infrastructure based network (where different quality of service traffic were continuously being sent by multiple users) was used to develop and validate Multiple User RTT models predicted from computed SNR.The models were also compared with single user models earlier developed. The tests results show that for multiple users on the network, RTT can be predicted from the computed SNR with reasonable accuracy as the models passed the $\mathrm{F}$ and $\mathrm{T}$ test at $0.1 \%$ level of significance and RMS errors of less than 867.378214ms were observed. During network design and installation, these models provide useful tools that can enable IEEE 802.11b WLAN installers to make fast and better informed RTT decisions.
\end{abstract}

\section{DOI: https://dx.doi.org/10.4314/jasem.v23i2.11}

Copyright: Copyright (C) 2019 Oghogho. This is an open access article distributed under the Creative Commons Attribution License (CCL), which permits unrestricted use, distribution, and reproduction in any medium, provided the original work is properly cited.

Dates: Received: 18 December 2018; Revised: 21 January 2019; Accepted 25 January 2019

Keywords: Round trip time, Signal to noise ratio, empirical models, Wireless Local Area Networks

WLANs are at the fore front in providing convenient access to the internet within organizations (Oghogho and Ezomo, 2013). Research has shown that throughput and RTT are the two most important metrics for determining WLANs performance (Geier, 2008a). A minimum throughput and a maximum RTT is necessary and must be satisfied by a network to ensure acceptable performance (Geier, 2008b). RTT has been defined as the time required for a signal pulse or packet to travel from a specific source to a specific destination and back again (Ali and Khuder; 2012). RTT depend on the throughput of the source connection and several other factors namely (El Miloud et al., 2013): (i) the nature of the transmission medium (ii) the physical distance between the source and the destination. (iii) the number of nodes between the source and the destination. (iv) the amount of traffic on the LAN (local area network) to which the end user is connected. (v) the number of other requests being handled by intermediate nodes and the remote server. (vi) the speed with which intermediate nodes and the remote server function. (vii) the presence of interference in the circuit. Most of these factors on which RTT depend (like the throughput of the source connection and factors (ii), (vi) and (vii) as listed above) are also directly related to the SNR present (Oghogho, 2018; Geier, 2008a; Geier, 2008b; Domenico and Stefan, 2011). Throughput in WLANs has been predicted directly from the computed SNR by Henty, (2001); Oghogho et al.,(2014a), Oghogho et al., (2014b) Oghogho et al.,(2015a), Oghogho et al.,(2015b), Oghogho, (2017), Oghogho et al., (2017), Oghogho et al.,(2018). Just as is the case for the throughput, several work including $\mathrm{Li}$ et al., (2009), El Miloud et al., (2013), Zobenko et al., (2014), Stephen, (2013), Nafei et al., (2013), Domenico and Stefan, (2011), Kavidha and Sadasivam (2010) exist that also studied and predicted the RTT. However, these researches do not directly model RTT from the observed SNR only. Oghogho (2018) showed that since RTT depends on several factors which are themselves directly dependent on the measured received signal strength indication from which SNR is computed, RTT can be modelled directly from the computed SNR for a single user on an IEEE802.11b WLAN. However no such work has been done for multiple users on the network. This paper seeks to fill this gap.

\section{MATERIALS AND METHODS}

The methods used in Oghogho, et al., (2014a) and Oghogho, (2018) were also used in this work except that multiple users RTT data was collected instead of Throughput data as done in Oghogho et al., (2014a). Also multiple users RTT data was collected instead of single user RTT data as done in Oghogho, (2018). The number of users was chosen as seven due to the work of $\mathrm{Wu}$ et al.,(2011) where seven users 
represented saturation traffic where each client always has a packet to send. Multiple Users RTT models predicted from SNR were statistically developed and compared with validation data for: (i) All signals considered, (ii) Strong signals (SNR $>25 \mathrm{~dB})$ only, (iii) Grey signals $(25 \mathrm{~dB}>\mathrm{SNR}>18 \mathrm{~dB})$ only (iv) Weak signals $(\mathrm{SNR}<19 \mathrm{~dB})$ only. The multiple users models developed were also compared with single user models earlier developed and their performances were tested by computing the RMS errors, " $F$ " and " $t$ " tests.

\section{RESULTS AND DISCUSSION}

Multiple Users RTT field and validation data statistical parameters are shown in Table 1 for different SNR categories. In Fig. 1, averages and standard deviation of multiple users RTT field data have been plotted against SNR. From Table 1, it can be observed that the standard deviation is high even when signal was strong implying that RTT varies considerably for multiple users on the network. This is a deviation from what was observed for single user on the network as reported by Oghogho (2018) where the observed RTT standard deviation was low.The increase in standard deviation at the boundary $(\mathrm{SNR}=25 \mathrm{~dB})$ between strong and grey signals can be noticed on Figure 1. For the RTT multiple users data statistics presented in this work, greater means, standard deviations and variances for RTT were observed for all signal ranges compared with single user data statistics presented by Oghogho (2018).

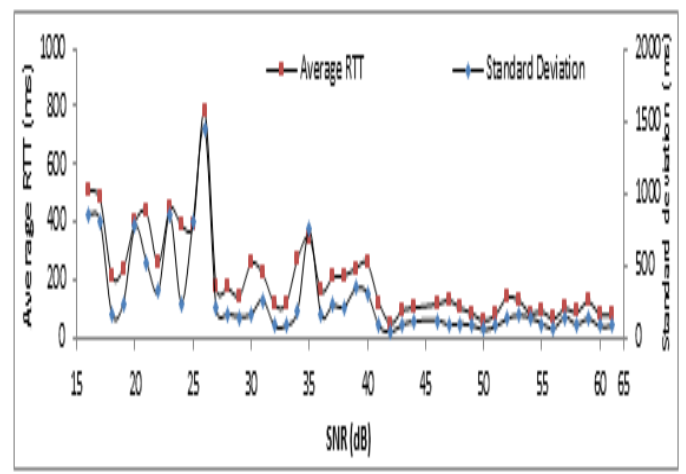

Fig. 1: Averages and Standard deviation of Multiple Users RTT field data Vs SNR.

Table 1: Statistical Parameter Values of RTT data for Different Cases of Received SNR.

\begin{tabular}{|c|c|c|c|c|}
\hline \multirow[t]{2}{*}{ Statistical Parameter } & \multicolumn{2}{|c|}{$\begin{array}{l}\text { ALL RSSI (SNR) considered } \\
(63 \mathrm{~dB} \geq S N R \geq 13 \mathrm{~dB})\end{array}$} & \multicolumn{2}{|c|}{ Strong Signal $(\mathrm{SNR} \geq 25 \mathrm{~dB})$} \\
\hline & $\begin{array}{l}\text { RTT Field } \\
\text { data }\end{array}$ & $\begin{array}{l}\text { RTT Validation } \\
\text { data }\end{array}$ & RTT Field data & $\begin{array}{l}\text { RTT Validation } \\
\text { data }\end{array}$ \\
\hline N (Sample Size) & 1844 & 524 & 1451 & 419 \\
\hline Mean & 191.96329 & 173.2341 & 148.437147 & 181.7918 \\
\hline Std. Error of Mean & 9.405635 & 18.03244 & 8.4579804 & 21.33340 \\
\hline Median & 93 & 40.5500 & 78.9 & 43.8000 \\
\hline Mode & 2.9 & 1.90 & 2.9 & 1.90 \\
\hline Std. Deviation & 403.8951 & 412.78149 & 322.1813368 & 436.68374 \\
\hline Variance & 163131.2 & 170388.559 & 103800.814 & 190692.691 \\
\hline Coefficient of dispersion & 2.1040226 & 2.3827958 & 2.17048996 & 2.4021091 \\
\hline Skewness & 7.442 & 5.589 & 10.331 & 5.592 \\
\hline Std. Error of Skewness & 0.057 & 0.107 & 0.064 & 0.119 \\
\hline Kurtosis & 72.012 & 39.193 & 135.857 & 37.950 \\
\hline Std. Error of Kurtosis & 0.114 & 0.213 & 0.128 & 0.238 \\
\hline Range & 5483.5 & 4161.80 & 5483.5 & 4161.80 \\
\hline \multirow{2}{*}{ Statistical Parameter } & \multicolumn{2}{|c|}{ Grey signal $(25 \mathrm{~dB}>\mathrm{SNR} \geq 19 \mathrm{~dB})$} & \multicolumn{2}{|c|}{ Weak Signal $($ SNR $<19$ dB $)$} \\
\hline & $\begin{array}{l}\text { RTT Field } \\
\text { data }\end{array}$ & $\begin{array}{l}\text { RTT Validation } \\
\text { data }\end{array}$ & RTT Field data & $\begin{array}{l}\text { RTT Validation } \\
\text { data }\end{array}$ \\
\hline N (Sample Size) & 362 & 39 & 32 & 66 \\
\hline Mean & 351.6677 & 183.4128 & 459.9094 & 112.8909 \\
\hline Std. Error of Mean & 30.82918 & 64.58314 & 143.8395 & 26.21066 \\
\hline Median & 177.2 & 23.8000 & 223.3 & 20.1500 \\
\hline Mode & 7.1 & $3.50^{*}, 7.5^{*}$ & 89.3 & $5.00^{*}, 6.2 *$ \\
\hline Std. Deviation & 586.56516 & 403.32160 & 813.6792 & 212.93640 \\
\hline Variance & 344058.683 & 162668.316 & 662073.8 & 45341.909 \\
\hline Coefficient of dispersion & 1.6681987 & 2.1988928 & 1.769216 & 1.886214 \\
\hline Skewness & 4.497 & 2.878 & 2.857 & 4.227 \\
\hline Std. Error of Skewness & 0.128 & 0.378 & 0.414 & 0.295 \\
\hline Kurtosis & 27.134 & 8.611 & 7.299 & 22.616 \\
\hline Std. Error of Kurtosis & 0.256 & 0.741 & 0.809 & 0.582 \\
\hline Range & 5038.40 & 1889.10 & 3161.8 & 1419.40 \\
\hline
\end{tabular}

This implies that packet queuing and delays in packet transmission increase with increase in the number of users on the network. This results because the network traffic is congested by the many users all 
having a packet to send. This congestion leads to the selection of lower transmission rates by the error control mechanism which aims to reduce errors in packet transmission thus leading to longer round trip times of packets.

Development of RTT Models: Parameters of Multiple users RTT models developed using Statistical packages for Social sciences (SPSS) are shown in Table 2. RTT (ms) is predicted directly from the observed SNR (dB). The four models (i) RTT General Multiple Users model (RTTGMUM), (ii) RTT Strong Signal Multiple Users Model, (RTTSSMUM) (iii) RTT Grey Signal Multiple Users Model (RTTGSMUM)and (iv) RTT Weak Signal Multiple Users Model (RTTWSMUM) are presented in equation 1-4 respectively. The model coefficient $\left(a_{1}\right)$ has different values for each model.

Table 2: Developed Models Parameters

\begin{tabular}{lllllll}
\hline S/N & Model Name & $\begin{array}{l}\text { Model } \\
\text { Description }\end{array}$ & $\begin{array}{l}\text { Sample } \\
\text { Size }\end{array}$ & $\begin{array}{l}\mathrm{R}^{2} \\
\text { value }\end{array}$ & $\begin{array}{l}\text { Standard error } \\
\text { of estimate }\end{array}$ & $\begin{array}{l}\text { Level of } \\
\text { significance }\end{array}$ \\
\hline 1 & RTTGMUM & Power Model & 1813 & 0.853 & 1.733 & $0.000 \%$ \\
2 & RTTSSMUM & Power Model & 1447 & 0.867 & 1.583 & $0.000 \%$ \\
3 & RTTGSMUM & Power Model & 334 & 0.920 & 1.466 & $0.000 \%$ \\
4 & RTTWSMUM & S-Curve Model & 32 & 0.953 & 1,215 & $0.000 \%$ \\
\hline
\end{tabular}

RTTGMUM $=f(S N R)=S N R^{a_{1}} \ldots \ldots \ldots \ldots \ldots 1$

RTTSSMUM $=f(S N R)=S N R^{a_{1}} \ldots \ldots \ldots \ldots \ldots \ldots . .2$

RTTGSMUM $=f(S N R)=S N R^{a_{1}} \ldots \ldots \ldots \ldots \ldots \ldots . . . . . .3$

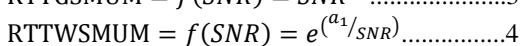

Tests and Discussion: To test the models, the Null hypothesis 1; $\left(\mathbf{H}_{\mathbf{0}}\right)$ was defined to mean RTT does not depend significantly on SNR when there are multiple Users on the network. Alternative hypothesis 1; HI was defined to mean RTT depends significantly on SNR when there are multiple Users on the network. Table 3 shows the root means square
(RMS) errors, the F-distribution and $\mathrm{T}$ test results. $\mathrm{H}_{0}$ was rejected for all cases and all the models were accepted at $1 \%$ level of significance at the respective degrees of freedom. This implies that for multiple users on the IEEE 802.11b WLAN, RTT significantly depends on SNR computed and the models developed can predict RTT from computed SNR within reasonable accuracy. Table 4 shows comparison of the multiple users RTT models developed in this work with the similar single user RTT models developed by Oghogho (2018).

\begin{tabular}{|c|c|c|c|c|c|c|c|}
\hline \multirow{2}{*}{$\begin{array}{l}\text { Model } \\
\text { type }\end{array}$} & \multicolumn{2}{|c|}{ RMS error value (ms) } & \multicolumn{2}{|c|}{$F$ test } & \multicolumn{2}{|c|}{ T Test } & \multirow[t]{2}{*}{ Decision } \\
\hline & & & $\begin{array}{l}\text { F value } \\
\text { from Model }\end{array}$ & $\begin{array}{l}\text { F value } \\
\text { from } \\
\text { Table }\end{array}$ & $\begin{array}{l}\text { T value } \\
\text { from } \\
\text { Model }\end{array}$ & $\begin{array}{l}\text { T value } \\
\text { from Table }\end{array}$ & \\
\hline \multirow[t]{4}{*}{$\begin{array}{l}\text { RTTGMU } \\
\text { M }\end{array}$} & All SNR & 186.604172 & $\begin{array}{l}\mathrm{F}_{0.01,1,1812}= \\
10531.945\end{array}$ & 6.63 & 70.295 & $\begin{array}{l}\mathrm{T}_{0.005,1884}= \\
2.58\end{array}$ & $\begin{array}{l}\mathrm{H}_{0} \text { is rejected. Model is } \\
\text { accepted at } 1 \% \text { level of }\end{array}$ \\
\hline & $\begin{array}{l}\text { Limited to Strong } \\
\text { Signals only }\end{array}$ & 186.1866975 & & & & & Significance \\
\hline & $\begin{array}{l}\text { Limited to Grey } \\
\text { Signals only }\end{array}$ & 247.809 & & & & & \\
\hline & $\begin{array}{l}\text { Limited to Weak } \\
\text { Signals only }\end{array}$ & 113.96176 & & & & & \\
\hline $\begin{array}{l}\text { RTTSSM } \\
\text { UM }\end{array}$ & 198.5662945 & & $\begin{array}{l}\mathrm{F}_{0.01,1,1446}= \\
9405.073\end{array}$ & 6.63 & 96.689 & $\begin{array}{l}\mathrm{T}_{0.005,1502}= \\
2.58\end{array}$ & $\begin{array}{l}\mathrm{H}_{0} \text { is rejected. Model is } \\
\text { accepted at } 1 \% \text { level of } \\
\text { Significance }\end{array}$ \\
\hline $\begin{array}{l}\text { RTTGSM } \\
\text { UM }\end{array}$ & 176.4396 & & $\begin{array}{l}\mathrm{F}_{0.01,1,333}= \\
3807.053\end{array}$ & 6.63 & 26.861 & $\begin{array}{l}\mathrm{T}_{0.005,315}= \\
2.58\end{array}$ & $\begin{array}{l}\mathrm{H}_{0} \text { is rejected. Model is } \\
\text { accepted at } 1 \% \text { level of } \\
\text { Significance }\end{array}$ \\
\hline $\begin{array}{l}\text { RTTWSM } \\
\text { UM }\end{array}$ & 867.378214 & & $\begin{array}{l}\mathrm{F}_{0.01,1,31}= \\
633.849\end{array}$ & 7.56 & 14.624 & $\begin{array}{l}\mathrm{T}_{0.005,62}= \\
2.62\end{array}$ & $\begin{array}{l}\mathrm{H}_{0} \text { is rejected. Model is } \\
\text { accepted at } 1 \% \text { level of } \\
\text { Significance }\end{array}$ \\
\hline
\end{tabular}

From Table 4, it can be seen that the RTTGMUM performed better than all others as it showed lower RMS errors in the different cases considered except for the grey signals where the RTTGSMUM (RMS error =176.4396) performed better than the RTTGMUM (RMS error =247.809). Also, all the Multiple users models developed in this work showed lower RMS errors compared with single user models developed by Oghogho 2018. However when the signal becomes weak, the multiple user models (developed from the weak signals field data) showed a very high RMS error (867.378214) compared with the RMS error (124.5541) of the corresponding single user models developed by Oghogho (2018). 


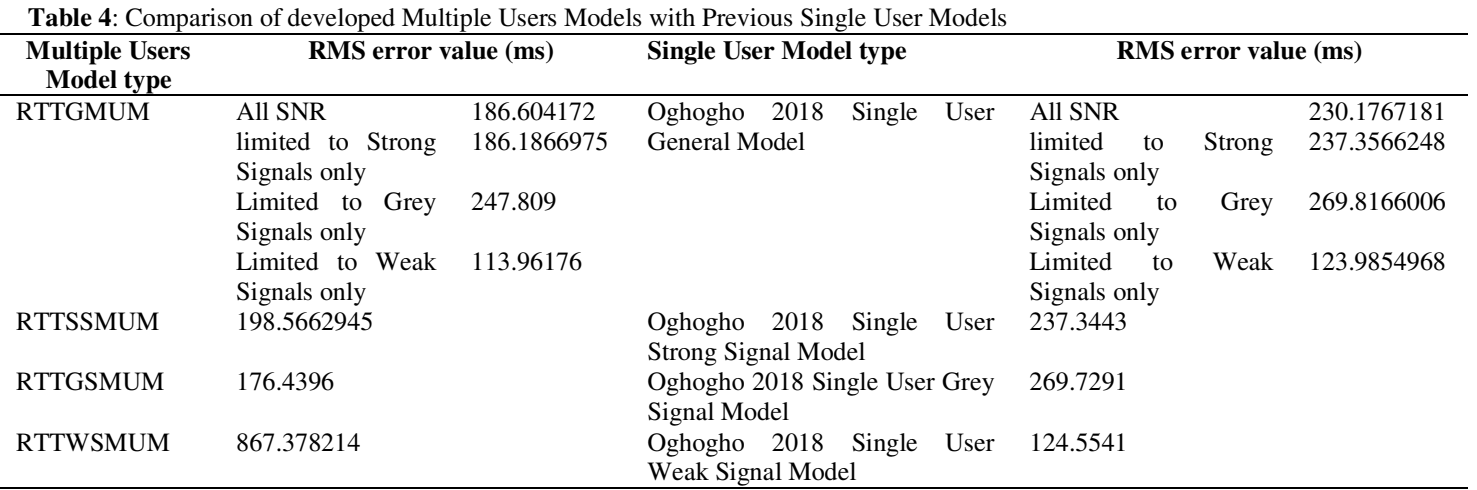

This happened because for multiple users, when signal has become weak the variability of RTT becomes very high as seen in the high standard deviation (813.6792ms) computed from the weak signal RTT field data statistics in Table 1. Thus for weak signals, the general model (RTTGMUM) should be used for RTT prediction. Figure 2 shows the plot of RTT validation data along with the General RTT multiple users model (RTTGMUM) and Oghogho 2018 Single User General RTT model. The graph shows clearly that the RTTGMUM developed in this work follows the validation data more closely. This was already proven from the computed RMS error in Table4. Figure 3-5 shows plots of RTT validation data along with the developed models for strong, grey and weak signals respectively.

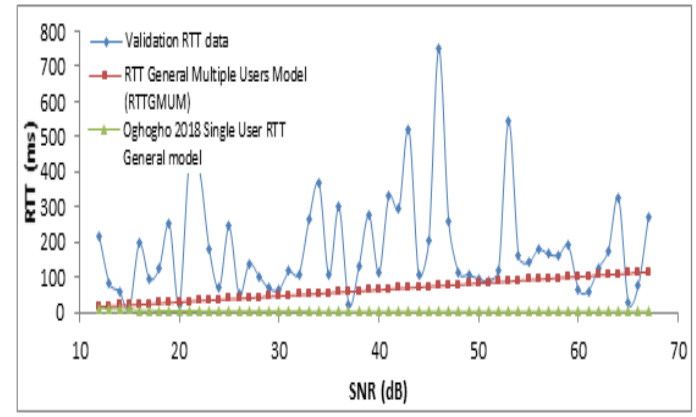

Fig. 2:Comparison of RTT Models for all SNR Considered

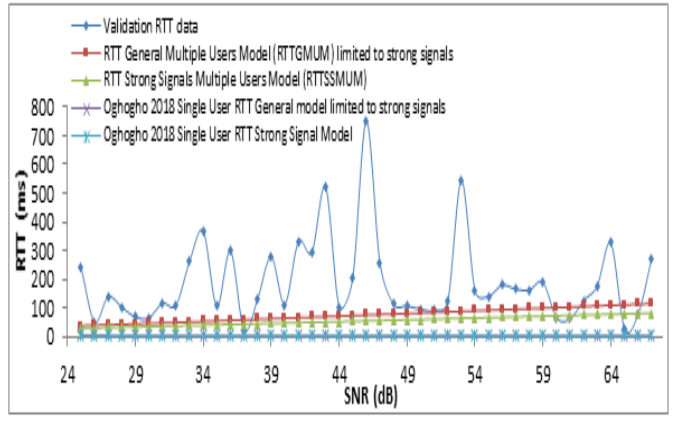

Fig.3: RTT Models in the Strong Signal Range only

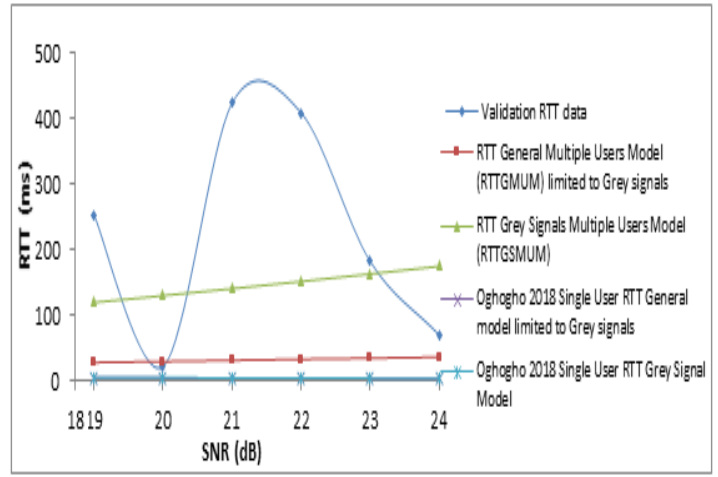

Fig.4: RTT Models in the Grey Signal Range only

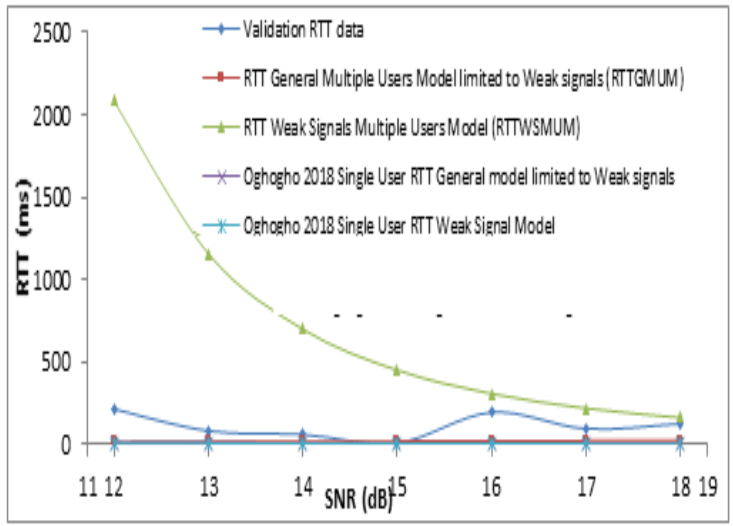

Fig.5: RTT Models in the Weak Signal Range only

From the various graphs of Figure 2-5 and Table 4 which compared the performances of the models using RMS errors, it can be seen that proceeding to develop models to predict RTT for multiple users in an IEEE 802.11b WLAN was necessary as the multiple users models performed better by showing lower RMS errors compared with that of the single user models earlier developed.

Conclusion: RTT multiple users models that can predict RTT based on the computed SNR only for 
various signal ranges in IEEE 802.11b WLANs have been developed, validated, tested for performance and compared with similar single user models earlier developed. By showing low RMS errors and having passed the $\mathrm{F}$ and $\mathrm{T}$ tests, the developed models can be relied upon to provide prediction of the RTT in IEEE 802.11b WLANs based on SNR observed.

Acknowledgement:The author acknowledges the contributions of Prof F.O Edeko and Prof J.O Emagbetere.

\section{REFERENCES}

Domenico D., Stefan M., (2011). CAESAR: Carrier Sense-Based Ranging in Off-The-Shelf802.11 Wireless LAN. ACM CoNEXT 2011, December 6-9 2011, Tokyo, Japan, pp 1-12

El Miloud A R., Kamal G., Koutaiba A., Otman E M., Slimane M.,(2013) Performance Analysis of Round Trip Delay Time in Practical Wireless Network for Telemanagement. World Academy of Science, Engineering and Technology. International Journal of Electrical, Robotics, Electronics and Communications Engineering Vol:7 No:11, pp 933-939, 2013

Geier. J., (2008a). Wi-Fi: Define Minimum SNR Values for Signal Coverage. Enterprise Networking Planet. Retrieved 4/04/13 at http://www.enterprisenetworkingplanet.com/nets p/article.php/3747656/WiFi-Define-Minimum$\underline{\text { SNR-Values-for-Signal-Coverage.htm }}$

Geier J., (2008b) How to: Conduct a Wireless Site Survey. Wi-fi Planet. Retrieved 29/07/14 at http://www.wifiplanet.com/tutorials/article.php/3761356

Kavidha V., Sadasivam V., (2010) Exploring Round Trip Time fairness for AdaptiveLayered Transmission Control Protocol. Int. J. of $A d v$. Networking and Applications. 1, (6) 353-358

Li H., Xiong N., Park JH., Cao, Q. (2009) Predictive control for vehicular sensor networks based on round-trip-time-delay prediction. IETCommun., 2010, Vol. 4, Iss. 7, pp. 801-809, ISSN 17518628. doi: 10.1049/iet-com.2009.0132

Nafei Z., Jingsha H., Yue Z., Wei W., (2013) On the Accuracy of Packet Delay Estimation in Distributed Service Networks. J NetwSyst Manage (2013) 21:623-649. DOI $10.1007 / \mathrm{s} 10922-013-9266-4$
Oghogho I., (2018). Single user round trip time models in IEEE $802.11 \mathrm{~b}$ WLANS. J. Res. Innov.Engineer. 3 (2) 24-29.

Oghogho I., Edeko, F O., Emagbetere, J. E (2018). Measurement and Modeling of TCP Downstream Throughput Dependence on SNR in an IEEE 802.11b WLAN System. J. King Saud Univ. -Engineer. Sci. 30: 170-176.

Oghogho I. (2017) Throughput dependence on SNR in IEEE 802.11 WLAN systems. In Ed. Mehdi Khosrow-Pour, Encyclopedia of Informations Science and Technology, Fourth Edition. IGI Global USA. pp 6618-6629; DOI: 10.4018/9781-5225-2255-3.ch574, ISBN 9781522522553 (set : hardcover) । ISBN 9781522522560 (ebook).

Oghogho I., Edeko, F O., Emagbetere, J. E. (2017). Empirical Investigation on the Dependence of TCP Downstream Throughput on SNR in an IEEE802. 11b WLAN System. Journal of King Saud University-Engineering Sciences. Vol (2017) 29: 135-143. DOI: http://dx.doi.org/10.1016/j.jksues.2015.06.006

Oghogho I., Edeko, F O., Emagbetere, J. E. (2015a) Probability Models for Predicting TCP Upstream Throughput in an IEEE802.11b WLAN System. J. Elect. Electronic Engineer. 12 (2) 1-19.

Oghogho I., Edeko, F O., Emagbetere, J. E, (2015b) Investigation on the Dependence of TCP Upstream Throughput on SNR For Single and Multiple Links in a WLAN System. Review of Information Engineering and Applications, 2015, 2(1): 15-32. Online ISSN: 2409-6539. DOI: 10.18488/journal.79/2015.2.1/79.1.15.32.

Oghogho, I., Edeko, F O., Emagbetere, J. E., \& Victor, M. (2014a). Empirical investigation on the dependence of TCP upstream throughput on SNR in an IEEE802. 11b WLAN system. In Telecommunications (ICT), $2014 \quad 21$ st International Conference on (pp. 442-446). IEEE. Doi:10.1109/ICT.2014.6845155

Oghogho I., Edeko, F O., Emagbetere, J. E (2014b). Empirical Probability Models for Predicting TCP Downstream throughput in a WLAN System. ISTP Journal of Research in Electrical and Electronics Engineering (ISTP-JREEE). Special issue. Pp 38-48. Available online at http://www.istp.org.in/Spec.Issue.html. 
Oghogho, I., Ezomo, P I., (2013). ICT for national development in Nigeria: creating an enabling environment. Int. J. Eng. Appl. Sci., 2305-8269 Vol3 (2), 59-66.

Stephen D F., (2013) PassivelyMeasuring TCPRound-TripTimes. Communications of the ACM October 2013, vol. 56, no. 10, doi:10.1145/2507771.2507781
Wu F., Tang B., Liu Y., Zhang L. (2011) Throughput Model of IEEE 802.11 DCF Considering MultiRate. Procedia Environmental Sciences 11 (2011) pp493-498.

Zobenko A., Scherrer T., Soo-Yong K. Proximity estimation for location-based services with round-trip time. ELECTRONICS LETTERS 3rd July 2014 Vol. 50 No. 14 pp. 1029-1031. 